\title{
The Green's function BEM for bimaterial solids applied to edge stress concentration problems
}

\author{
M. Denda \\ Rutgers University, Mech. \& Aero. Engng. Dept. \\ Piscataway, NJ 08854-8058, U.S.A.
}

\begin{abstract}
A boundary element method (BEM) for bimaterial domains consisting of two isotropic solids bonded perfectly along the straight interface will be developed. We follow the physical interpretation of Somigliana's identity to represent the displacement in the bimaterial domain by the continuous distributions of the line forces and dislocation dipoles over its boundary. The fundamental solutions used are the Green's functions for the line force and the dislocation dipole that satisfy the traction and displacement continuity across the interface of two domains. There is no need to model the interface because the required continuity conditions there are automatically satisfied by the Green's functions.

The BEM will be applied to study the edge stress concentration of the bimaterial solids. We calculate the singular stress distribution at the free edge of the interface for various bimaterial configurations and loadings, in particular, for the domain consisting of thin coating over the substratum. Since the Green's function BEM does not require the boundary elements on the interface, it can handle the edge singularity on the interface accurately even for extremely thin coatings. The BEM developed here is not limited to the edge stress concentration problems and can be applied to a broad range of the bimaterial domain problems effectively.
\end{abstract}

\section{Introduction}

We explore the use of the special fundamental solutions, in the boundary element method (BEM), that satisfy the continuity of the displacement and traction along the straight interface of perfectly bonded two dissimilar isotropic half-planes in two-dimensions. Such fundamental solutions are called the Green's functions and the boundary element method using the Green's functions as the Green's function BEM. For the half-plane problems in two-dimensions the Green's functions that satisfy the traction-free boundary condition on the surface of the half-plane have been used by Telles and Brebbia [1] and Meek and Dai [2] for isotropic solids, Dumir and Mehta [3] for orthotropic solids, and Pan et al. [4] for general anisotropic solids to formulate the Greens function BEMs. The Greens functions that satisfy the crack surface traction-free boundary condition for a single crack have been used by Snyder and Cruse [5], Clements and Haselgrove [6], and Ang [7] for anisotropic solids. The Greens function BEMs for the domain containing an elliptical hole have been proposed by Morjaria and Mukherjee [8] and Denda and Kosaka [9] for the isotropic solids, Kamel and Liaw [10] and Hwu and Yen [11] for the anisotropic 
solids in plane strain/stress and generalized plane strain, respectively. For the bimaterial domain problems Berger [12] has obtained the Green's functions for the general anisotropic solids and formulated the bimaterial domain Green's function BEM. For interface crack problems, Yuuki abd Cho [13] and Berger and Tewary [14] have proposed the Green's function BEMs for the bimaterial isotropic solids and general anisotropic solids, respectively. In General the number of the Green's function BEMs for the elasticity problems is limited to problems with simple geometry due to the lack of the Green's functions for more complex problems as opposed to those in the potential type problems (Melnikov [15]).

The majority of the Green's functions available are two-dimensional and have been obtained by the complex variable methods in elasticity (Muskhelishvili [16], England [17]) where the complex displacement $u_{1}+i u_{2}$ is used in place of the real components $u_{1}$ and $u_{2}$, where $i=\sqrt{-1}$. These Green's functions, originally obtained in the complex variable form, are converted to the real variable form to be used for the standard BEMs. For two-dimensional problems we demonstrate that this conversion is unnecessary and present the Somigliana's identity in the complex variable form. This complex variable Somigliana's identity consists of complex valued boundary integrals that represent the continuous distributions of the complex valued line forces and line dislocation dipoles. We adopt the straight boundary element and perform these integrals analytically. It is after the analytical evaluation of these integrals that the complex form is converted to the real form to construct the standard system of boundary equations. The proposed BEM is equivalent to the standard direct formulation of the BEM.

Many composite materials consists of layers of bonded dissimilar materials. The debonding of these layers along the interface is most likely to occur at its free edge where the stress become concentrated. We apply the Green's function BEM to analyze the edge stress concentration of the bimaterial solids which was studied analytically by Bogy [18]. No previous BEM or the Green's function BEM has treated this problem. We calculate the singular stress distribution at the free edge of the interface for various bimaterial configurations and loadings, in particular, for the domain consisting of thin coating over the substratum. Since the Green's function BEM does not require the boundary elements on the interface, it can handle the edge singularity on the interface accurately even for extremely thin coatings. The BEM developed, not limited to the edge stress concentration problems, can be applied to a broad range of the bimaterial domain problems effectively.

\section{Somigliana's identity for the Green's function BEM}

Consider the line force applied at $\mathbf{x}$ in an infinite bimaterial plane $B^{\infty}$ with the straight interface. The resulting displacement and traction solutions at $\mathbf{y}$ that satisfy the continuity of the displacement and traction on the interface are called the Green's functions. For an arbitrary shaped finite bimaterial domain $B$, with 
the straight interface, subject to the traction $T_{\alpha}$ and displacement $U_{\alpha}$ on the boundary $\partial B$, the displacement is given by Somigliana's identity

$$
u_{\gamma}(\mathbf{x})=\int_{\partial B} T_{\alpha}(\mathbf{y}) G_{\alpha \gamma}(\mathbf{y}, \mathbf{x}) d s(\mathbf{y})-\int_{\partial B} U_{\alpha}(\mathbf{y}) G^{*}{ }_{\alpha \gamma}(\mathbf{y}, \mathbf{x}) d s(\mathbf{y}) \quad(\mathbf{x} \in B),
$$

where $G_{\alpha \gamma}(\mathbf{y}, \mathbf{x})$ and $G_{\alpha \gamma}{ }^{*}(\mathbf{y}, \mathbf{x})$ are the displacement and traction Green's functions, respectively. Notice that the integration is performed only over the boundary of the bimaterial domain and does not include the interface.

The line dislocation dipole is an infinitesimal segment $d s$ that undergoes a displacement jump. Let $G_{\alpha \gamma}(\mathbf{y}, \mathbf{x})^{*} d s$ be the $\alpha$ th displacement component at $\mathbf{y}$ when the unit $\gamma$ th component of the displacement jump is specified at $\mathbf{x}$ over $d s$ in $B^{\infty}$. Using the reciprocity relation

$$
G_{\alpha \gamma}(\mathbf{y}, \mathbf{x})=G_{\gamma \alpha}(\mathbf{x}, \mathbf{y})
$$

for the displacement Green's function, we can show the relation,

$$
-G^{*}{ }_{\alpha \gamma}(\mathbf{y}, \mathbf{x})=G_{\gamma \alpha}(\mathbf{x}, \mathbf{y})^{*},
$$

between the the traction Green's function of the line force and the displacement Green's function of the line dislocation dipole as shown by Denda and Kosaka [9]. By substituting the relations (2) and (3) into (1) we get an alternative form of Somigliana's identity

$$
u_{\gamma}(\mathbf{x})=\int_{\partial B} G_{\gamma \alpha}(\mathbf{x}, \mathbf{y}) T_{\alpha}(\mathbf{y}) d s(\mathbf{y})+\int_{\partial B} G_{\gamma \alpha}(\mathbf{x}, \mathbf{y})^{*} U_{\alpha}(\mathbf{y}) d s(\mathbf{y}),
$$

for $\mathbf{x} \in B$ and zero displacement outside $B$. According to the identity (4), the displacement field in the bimaterial body $B$ subject to the traction $T_{\alpha}$ and the displacement $U_{\alpha}$ on the boundary is given by the distributions of the line forces and dislocation dipoles, with the densities $T_{\alpha}$ and $U_{\alpha}$, respectively, over a contour $\partial B$, which now is simply a line marked out in the infinite bimaterial medium $B^{\infty}$. The two forms of Somigliana's identity, representing its mathematical (1) and physical (4) interpretations, are equivalent and either can be used for the direct formulation of the BEM. We adopt the latter form since it is amenable to the complex variable representation.

\section{Green's Functions}

\subsection{Line Force and Dislocation}

In Muskhelishvili's complex variable formalism ([16] and [17]) for isotropic elasticity we seek for the complex displacement $u=u_{x}+i u_{y}$ at point $z=x+i y$ resulting from the complex line force $f=f_{x}+i f_{y}$ or the line dislocation $b=b_{x}+i b_{y}$ at $\zeta=\xi+i \eta$. The components $\left(u_{x}, u_{y}\right),\left(f_{x}, f_{y}\right)$, and $\left(b_{x}, b_{y}\right)$ represent the displacement, line force and line dislocation (or Burgers) vectors, respectively. The 
displacement Green's functions for the line force and the dislocation solutions are closely related due to their duality relations (Ni and Nemat-Nasser [19]). A dislocation dipole consists of a pair of \pm dislocations separated by an infinitesimal distance. It represents a displacement jump along the infinitesimal line segment connecting the two dislocations and its solution is obtained from the dislocation solution by applying the total derivative operator. The Green's functions used as the kernel functions of the BEM are those for the line force and the dislocation dipole.

We consider an infinite bimaterial domain consisting of the upper and the lower halves, $R_{1}$ and $R_{2}$, bonded perfectly together along the straight interface. For the line force $f$ and line dislocation $b$ at $\zeta$ in $R_{1}$, we derive the displacement Green's functions by enforcing the continuity of the displacement and the traction along the interface. The standard framework of the complex variable methods in elasticity that uses a set of analytic functions, as summarized in Appendix A, yields the displacement Green's function solutions given by

$$
\begin{aligned}
2 \mu_{1}\left\{\left(u_{x}+\right.\right. & \left.\left.i u_{y}\right)_{1}-\left(u_{x}+i u_{y}\right)_{r b}\right\} \\
= & -\left\{\kappa_{1} \log (z-\zeta)-k_{1} \overline{\log (z-\zeta)}\right. \\
& \left.+\kappa_{1} k_{1} \delta_{1} \log (z-\bar{\zeta})-\lambda_{1} \overline{\log (z-\bar{\zeta})}+\delta_{1} \frac{(z-\bar{z})(\zeta-\bar{\zeta})}{\overline{(z-\bar{\zeta})})^{2}}\right\} \gamma_{1} \\
& +\left\{\frac{(z-\bar{z})-(\zeta-\bar{\zeta})}{\overline{z-\zeta}}+\delta_{1}\left(\kappa_{1} \frac{\zeta-\bar{\zeta}}{z-\bar{\zeta}}+k_{1} \frac{z-\bar{z}}{\overline{z-\bar{\zeta}}}\right)\right\} \bar{\gamma}_{1} \quad\left(z \in R_{1}\right), \\
2 \mu_{2}\left\{\left(u_{x}+\right.\right. & \left.\left.i u_{y}\right)_{2}-\left(u_{x}+i u_{y}\right)_{r b}\right\} \\
= & -\left\{\kappa_{2}\left(1+\lambda_{1}\right) \log (z-\zeta)-\left(1+\delta_{1}\right) k_{1} \overline{\log (z-\zeta)}\right\} \gamma_{1} \\
& +\frac{\left(1+\lambda_{1}\right)(z-\bar{z})-\left(1+\delta_{1}\right)(\zeta-\bar{\zeta})}{\overline{z-\zeta}} \bar{\gamma}_{1} \quad\left(z \in R_{2}\right)
\end{aligned}
$$

where

$$
\left\{\begin{array}{lll}
k_{1}=-\kappa_{1}, & \gamma_{1}=\frac{f}{2 \pi\left(\kappa_{1}+1\right)} & \text { (line force) } \\
k_{1}=1, & \gamma_{1}=\frac{i \mu_{1} b}{\pi\left(\kappa_{1}+1\right)} & \text { (line dislocation) }
\end{array}\right.
$$

and

$$
\kappa_{1}=3-4 \nu_{1} \quad(\text { plane strain }), \quad\left(3-\nu_{1}\right) /\left(1+\nu_{1}\right) \quad \text { (plane stress) }
$$

in terms of Poisson's ratio $\nu_{1}$. Further the coefficients $\delta_{1}$ and $\lambda_{1}$ are given by

$$
\delta_{1}=\frac{\alpha_{1}-\beta_{1}}{1+\beta_{1}}, \quad \lambda_{1}=\frac{\alpha_{1}+\beta_{1}}{1-\beta_{1}},
$$

in terms of Dundurs' parameters [20]

$$
\alpha_{1}=\frac{\mu_{2}\left(\kappa_{1}+1\right)-\mu_{1}\left(\kappa_{2}+1\right)}{\mu_{2}\left(\kappa_{1}+1\right)+\mu_{1}\left(\kappa_{2}+1\right)}, \quad \beta_{1}=\frac{\mu_{2}\left(\kappa_{1}-1\right)-\mu_{1}\left(\kappa_{2}-1\right)}{\mu_{2}\left(\kappa_{1}+1\right)+\mu_{1}\left(\kappa_{2}+1\right)},
$$


where $\kappa_{2}$ is defined by Poisson's ratio $\nu_{2}$ of material 2 by the relations similar to (8). Note that the subscripts 1 and 2 refer quantities associated with the regions $R_{1}$ and $R_{2}$, respectively. We use the principal branch for the logarithmic functions so that their arguments range from $-\pi$ (exclusive) to $+\pi$ (inclusive). The term $\left(u_{x}+i u_{y}\right)_{r b}$ is the rigid body displacement to be determined next.

If we apply a line force component $f_{y}$ at $\mathrm{P}(\zeta=\xi+i \eta)$ in $R_{1}$ (upper half), we expect the displacement component $u_{x}$ to vanish at the point $\mathrm{Q}\left(z_{0}=\xi\right)$ on the interface directly below the source $\mathrm{P}$ to satisfy the symmetry condition. Further, if we apply a force component $f_{x}$ at $\mathrm{Q}$ we expect the displacement $u_{y}$ at $\mathrm{P}$ to vanish to satisfy the reciprocity condition. However, the line force Green's function (5) does not satisfy these conditions until we add the complex rigid body displacement

$$
\left(u_{x}+i u_{y}\right)_{r b}=i \frac{\lambda_{1}-\kappa_{1}^{2} \delta_{1}}{8 \mu_{1}\left(\kappa_{1}+1\right) \pi^{2}}\left(f_{x}+i f_{y}\right)
$$

in both $R_{1}$ and $R_{2}$. Notice that if we replace $\log (z-\bar{\xi})$ in (5) with $\log (z-\bar{\xi})-\log (i)$, then the additional term $-\log (i)$ will produce the rigid body displacement (11). The rigid body displacement for the line dislocation is set to zero. For the line force $f$ and line dislocation $b$ at $\zeta$ in $R_{2}$ (lower half-plane), the Green's function solution is obtained if we swap indices 1 and 2 in equations (5)-(11) and replace $i$ by $-i$ in (11).

\subsection{Dislocation Dipole}

Consider a pair of line dislocations with the Burgers vectors $-b$ and $b$ located at $\zeta$ and $\zeta+d \zeta$, respectively. This pair produces a displacement jump over the infinitesimal line segment $d \zeta$ connecting the two dislocation sites and called a dislocation dipole. Its Green's function is obtained by applying the total differential operator,

$$
d=\frac{\partial}{\partial \zeta} d \zeta+\frac{\partial}{\partial \bar{\zeta}} d \bar{\zeta}
$$

to the Green's function (5) and (6) of the dislocation.

\section{Boundary Equations}

\subsection{Constant Interpolation}

We approximate the boundary by a set of straight lines $L_{j}(j=1,2, \ldots, m)$ with the slope $\phi_{j}$ and use the constant interpolation for the boundary traction $T_{j}=T_{j x}+i T_{j y}$ and displacement $U_{j}=U_{j x}+i U_{j y}$ for each element $L_{j}$. There is no need to introduce boundary elements along the interface since the interface is built in the Green's functions. We evaluate the displacements at the middle points $z_{k}(k=1,2, \ldots, m)$ of the boundary elements to set up the system of boundary 
equations,

$$
2 U_{k}=\sum_{j=1}^{m}\left[M_{k j} T_{j}+\bar{N}_{k j} \bar{T}_{j}\right]-\sum_{j=1}^{m}\left[K_{k j} U_{j}+\bar{L}_{k j} \bar{U}_{j}\right] \quad(k=1,2, \ldots, m),
$$

where the complex valued coefficients $M_{k j}, N_{k j}, K_{k j}$, and $L_{k j}$ are given in Appendix B. The actual system we solve is the real variable form of (13) given by

$$
\begin{aligned}
& 2\left\{\begin{array}{c}
U_{k x} \\
U_{k y}
\end{array}\right\}= \sum_{j=1}^{m}\left[\begin{array}{ll}
\Re\left(M_{k j}+N_{k j}\right), & -\Im\left(M_{k j}+N_{k j}\right) \\
\Im\left(M_{k j}-N_{k j}\right), & \Re\left(M_{k j}-N_{k j}\right)
\end{array}\right]\left\{\begin{array}{c}
T_{j x} \\
T_{j y}
\end{array}\right\} \\
&- \sum_{j=1}^{m}\left[\begin{array}{ll}
\Re\left(K_{k j}+L_{k j}\right), & -\Im\left(K_{k j}+L_{k j}\right) \\
\Im\left(K_{k j}-L_{k j}\right), & \Re\left(K_{k j}-L_{k j}\right)
\end{array}\right]\left\{\begin{array}{c}
U_{j x} \\
U_{j y}
\end{array}\right\} \\
&(k=1,2, \ldots, m),
\end{aligned}
$$

where $\Re$ and $\Im$ indicate the real and the imaginary parts. This system (14) of the boundary algebraic equations is equivalent to the traditional system of the boundary integral equations in the direct formulation except that the coefficients of the former are evaluated analytically and no integrals are involved.

\subsection{Higher order interpolation}

The use of higher order interpolation functions is essential in the analyses of bimaterial plates where stress concentration is expected near the edges of the interface as indicated by Bogy [18]. We discretize the boundary by straight elements and approximate the boundary displacement and traction by the quadratic functions. The boundary integrals are evaluated analytically. The essence of the BE implementation remains the same as the case of the constant interpolation and the details are omitted.

\section{$5 \quad$ Edge Stress Concentration Analysis}

Note that all the numerical results in this section have been obtained using the quadratic interpolation functions. First we have analyzed a square plate in uniaxial tension ( $\sigma_{0}$ in $x_{2}$ direction) using one of the material combinations chosen by Bogy [18]: $\nu_{1}($ material 1$)=\nu_{2}($ material 2$)=0.5$ with the ratio of shear modulus $\mu_{2} / \mu_{1}=0.111$ or $\alpha_{1}=-0.8$ and $\beta_{1}=0$ in terms of Dundurs' parameters defined by (10). Thickness of the two material layers are equal and plane strain is assumed. Number of elements introduced along the plate boundary, which are progressively refined as the interface is approached, is 32 when the entire plate is analyzed. The size of the smallest boundary element, which appear adjacent to the interface, is $1 / 100000 t h$ of the half plate width $w$. Since stress component $\sigma_{11}$ is expected to be discontinuous across the interface the stress calculation cannot be performed exactly on the interface; rather it is performed along two horizontal 

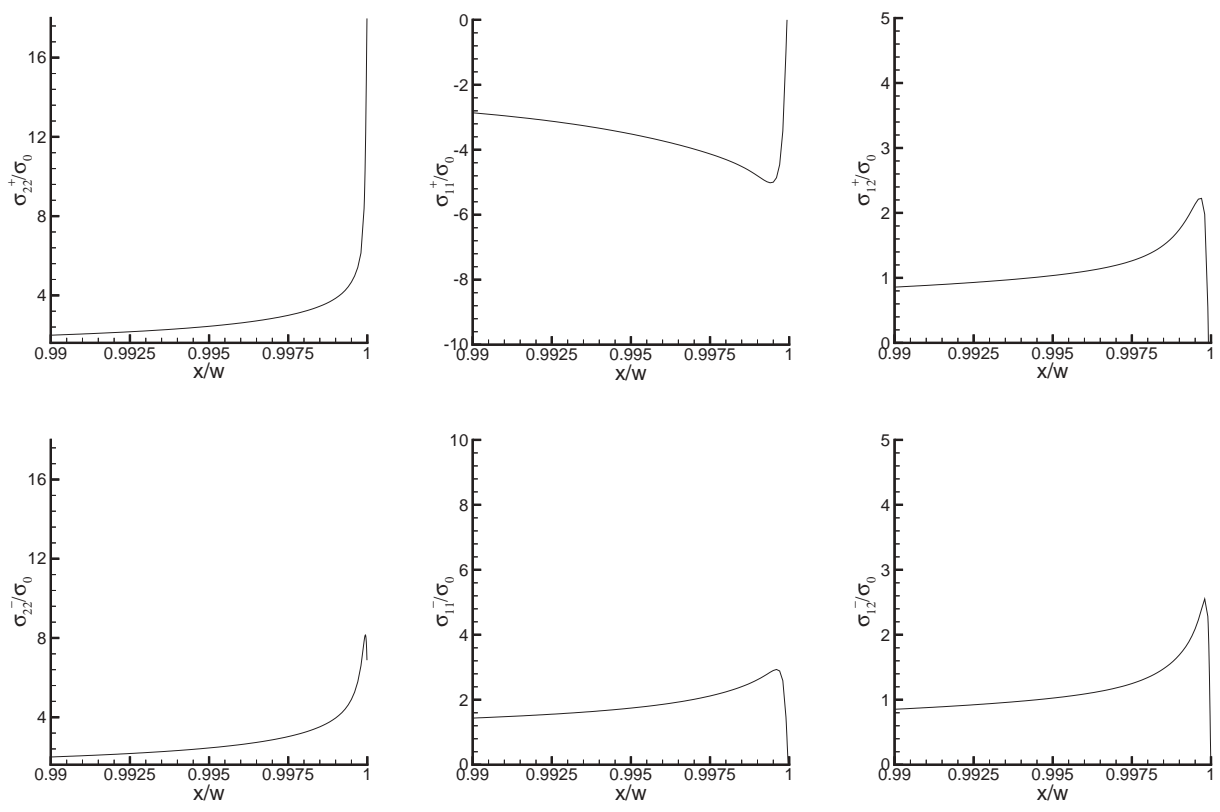

(a) $\sigma_{22}{ }^{+}$and $\sigma_{22}$

(b) $\sigma_{11}{ }^{+}$and $\sigma_{11}$

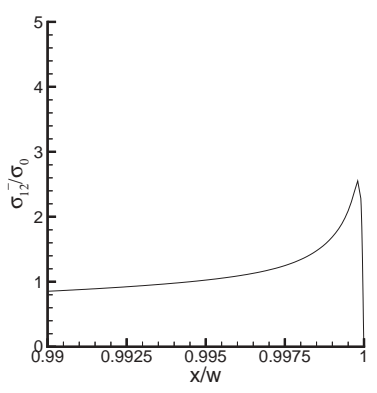

(c) $\sigma_{12}{ }^{+}$and $\sigma_{12}$

Figure 1: Bimaterial square plate $\left(\mu_{2} / \mu_{1}=0.111, \nu_{1}=\nu_{2}=0.5\right)$ in tension. Variation of the stress along horizontal lines $y / w= \pm 1.0 \times 10^{-4}$ in the immediate neighborhood of the interface edge. (a) $\sigma_{22}^{ \pm}$, (b) $\sigma_{12}^{ \pm}$, and (c) $\sigma_{11}^{ \pm}$. Superscripts \pm indicate evaluation at $\pm 1.0 \times 10^{-4}$. Thickness of the two layers are the same.

lines $y / w= \pm \epsilon$ just above and below the interface. For calculations of the interface stress, the offset distance $\epsilon$ should be small enough so that the values of the stress components $\sigma_{22}$ and $\sigma_{12}$ evaluated at two adjacent points across the interface are almost identical, which becomes increasingly difficult as we approach the extreme vicinity of the interface edge; the chosen values of $\epsilon$ for the interface stress calculation is $\epsilon= \pm 1.0 \times 10^{-4}$ and $\pm 1.0 \times 10^{-5}$. The results are shown in Fig. 1 (for $\epsilon= \pm 1.0 \times 10^{-4}$ ) and 2 (for $\pm 1.0 \times 10^{-5}$ ). The calculated values of the interface stress agree very well with those obtained by Bogy [18] near the interface in the range $0.5<r / w<1 / 1000$, where $r$ is the distance from the interface edge; note that Bogy has analyzed the infinite strip with finite width and that he has stopped the calculation at about $r / w=1 / 1000$. What is alarming is that as we calculate the interface stress nearer the edge than Bogy did (i.e., up to $1 / 100000$ of the plate width) the value of shear stress $\sigma_{12}$ (not the normal stress $\sigma_{22}$ which is known to be infinite) can be several times higher than Bogy predicted. Note that this shear stress drops suddenly to zero at the edge where the traction free boundary condition has to be satisfied. Further the value of normal stress 

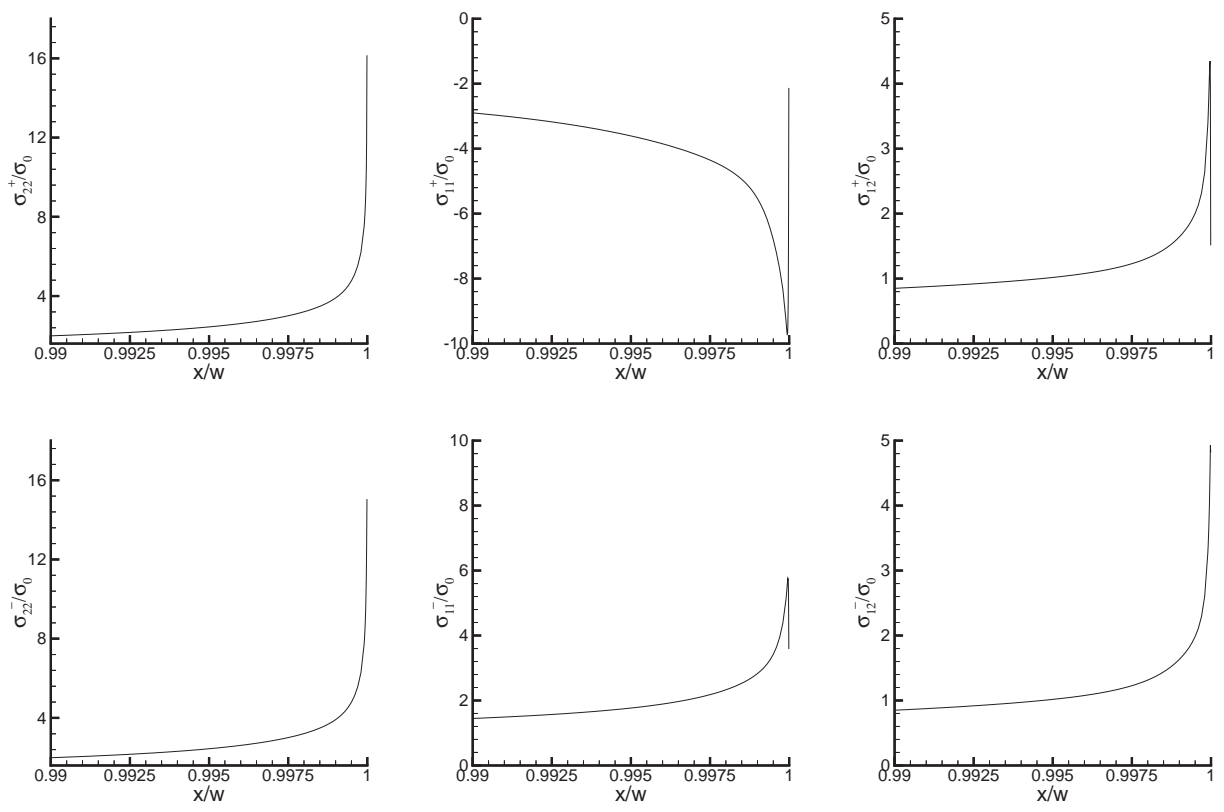

(a) $\sigma_{22}{ }^{+}$and $\sigma_{22}$

(b) $\sigma_{11}{ }^{+}$and $\sigma_{11}$

(c) $\sigma_{12}{ }^{+}$and $\sigma_{12}$

Figure 2: Bimaterial square plate $\left(\mu_{2} / \mu_{1}=0.111, \nu_{1}=\nu_{2}=0.5\right)$ in tension. Variation of the stress along horizontal lines $y / w= \pm 1.0 \times 10^{-5}$ in the immediate neighborhood of the interface edge. (a) $\sigma_{22}^{ \pm}$, (b) $\sigma_{12}^{ \pm}$, and (c) $\sigma_{11}^{ \pm}$. Superscripts \pm indicate evaluation at $\pm 1.0 \times 10^{-4}$. Thickness of the two layers are the same.

$\sigma_{11}$ can be as high as $\sigma_{11}=-10 \sigma_{0}$ (compression) for material 1 and $\sigma_{11}=+6 \sigma_{0}$ (tension) for material 2 at the interface. (Note that Bogy did not calculate $\sigma_{11}$.) The component $\sigma_{11}$ is discontinuous across the interface and drops suddenly to zero at the edge.

The issue of artificially high value of stress as boundary element nodes are approached has to be carefully taken care especially when we evaluate the interface stress. When constant interpolation functions are used the value of the stress, as a rule of thumb, is affected by the individual boundary element singularity within the disc (centered at an element end point) whose radius is approximately equal to the size of the element. Although this radius of influence due to the boundary element singularity is drastically reduced when quadratic interpolation functions are used, we have avoided evaluating the interfacial stress within the distance equal to the smallest size of the element which is located adjacent to the interface edge. Thus we are confident that the calculated value of the interface stress is not affected by the artificial singularity. Similar precautions should be applied for stress calculations at any points in the vicinity of the interface edge. 


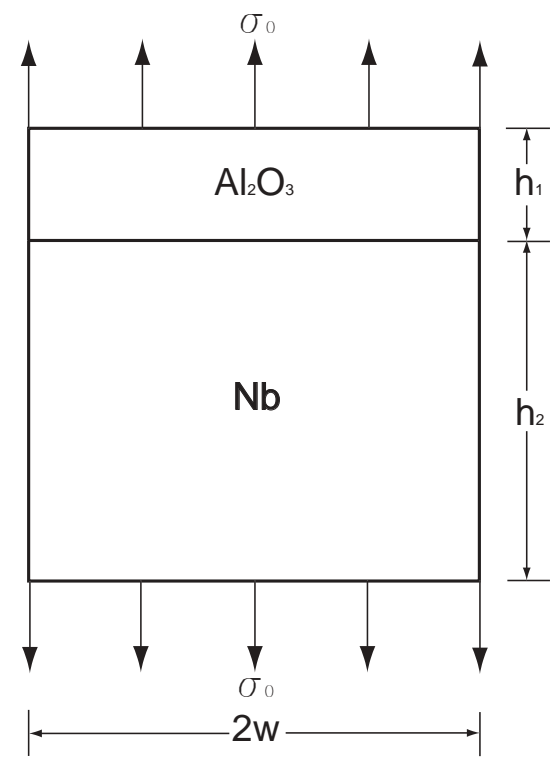

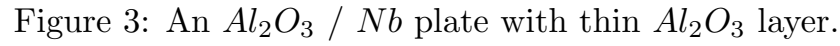

Next we consider $\mathrm{Al}_{2} \mathrm{O}_{3}$ (ceramic) / $\mathrm{Nb}$ (metal) bonded square plate in tension, as shown in Fig. $3\left(h_{1}=h_{2}=w\right)$, which represents more realistic material combination than selected by Bogy. Fig. 4 shows the interface stress distribution. This material combination characterized by Dundurs' parameters $\alpha_{1}=-0.52$ and $\beta_{1}=-0.57$ has been taken from Evans et al (1986). The mesh used is the same as the first example above and plane strain is assumed. Again the high stress concentration for the component $\sigma_{12}$ and, especially, $\sigma_{11}$ is observed as well as singular behavior for $\sigma_{22}$ at the edge. Although the finite element calculations by Evans et al [21] are qualitatively correct their values of interface stress are much lower than predicted in Fig. 4. The variation of the individual stress component in the entire plate is shown in Figs. 5, 7 and 9 in contour plots and 6, 8 and 10 in 3D plots. To see effects of the thickness $h$ of the $\mathrm{Al}_{2} \mathrm{O}_{3}$ layer we have analyzed the bimaterial plate with variable $\mathrm{Al}_{2} \mathrm{O}_{3}$ layer thickness $(h / w=1.0,0.1$, and 0.01) while fixing the thickness of the $N b$ layer as shown in Fig. 3. The interface stress components for various thickness are shown in Figs. 11 and 12. Note that for very thin layer of $\mathrm{Al}_{2} \mathrm{O}_{3}$ the distance between the interface, where the stress is evaluated, and the top edge of the plate, where boundary elements are located, becomes very small. In order that the calculated stress is not influenced by artificial boundary element singularities we have refined the boundary element mesh progressively as the thickness of the layer has been reduced: 32,54 , and 66 elements for $h / w=1.0$, 0.1 , and 0.01 , respectively. In particular in producing the solution curves for the layer thickness $h / w=0.01$, which are accompanied by extremely sharp spikes near the edge, we have used several different boundary element meshes to make sure 

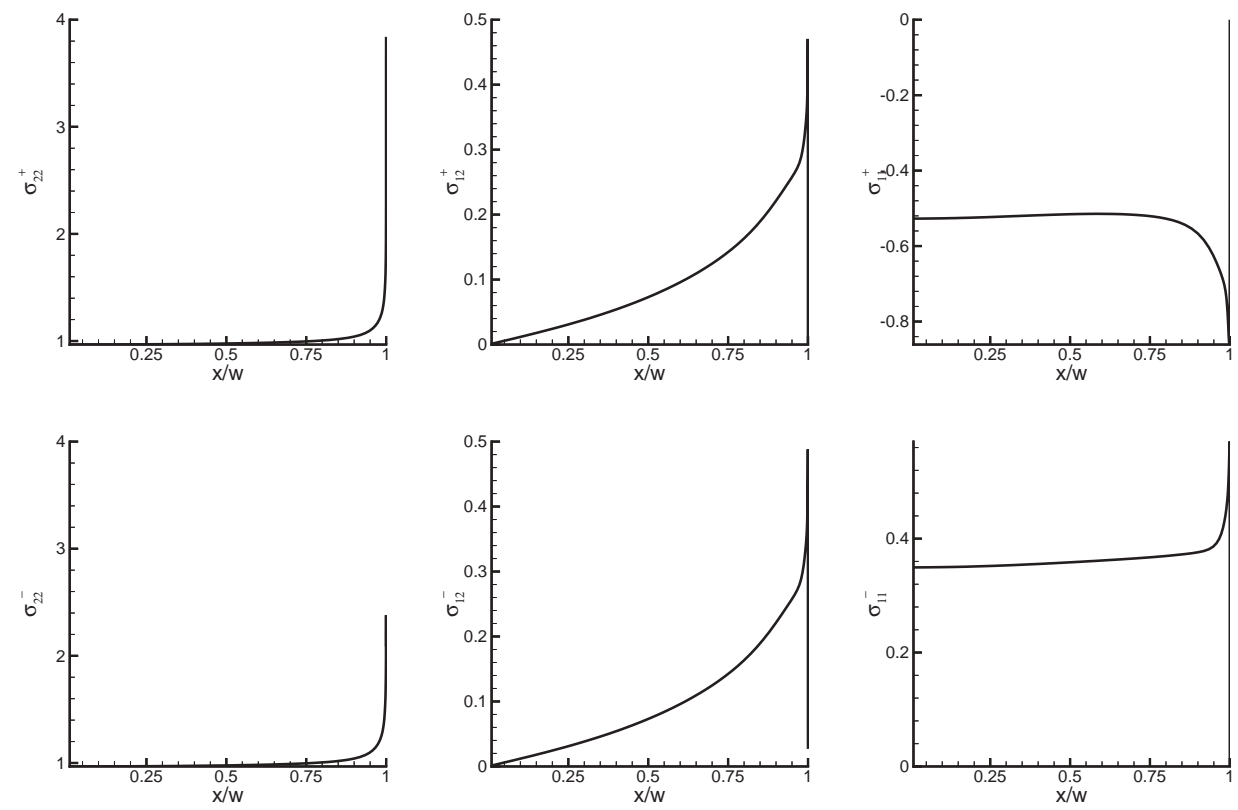

(a) $\sigma_{22}{ }^{+}$and $\sigma_{22}$

(b) $\sigma_{11}{ }^{+}$and $\sigma_{11}$

(c) $\sigma_{12}{ }^{+}$and $\sigma_{12}$

Figure 4: Interface stress for an $\mathrm{Al}_{2} \mathrm{O}_{3} / \mathrm{Nb}$ square plate in tension. Variation of the stress along horizontal lines $y / w= \pm 1.0 \times 10^{-3}$ in the immediate neighborhood of the interface edge. (a) $\sigma_{22}^{ \pm}$, (b) $\sigma_{12}^{ \pm}$, and (c) $\sigma_{11}^{ \pm}$. Superscripts \pm indicate evaluation at $\pm 1.0 \times 10^{-3}$. Thickness of the two layers are the same.

that these are not influenced by the artificial singularities. Notice that when the thickness is reduced degree of the stress concentration is somewhat reduced near the edge for all the components. However, when $h / w=0.01$ the magnitude of the compressive stress $\sigma_{11}$ in the thin $\mathrm{Al}_{2} \mathrm{O}_{3}$ layer increases throughout the entire thin layer which could trigger buckling of this layer upon the development of debonding at the interface edge or could cause extensive plastic deformation, not only near the edge, but throughout the layer. The presence of this high compressive stress $\sigma_{11}$, for the thin layer whose shear modulus is higher than that of the stratum, has not been reported in the past and we believe is an additional important factor in explaining various fracture mechanisms. Figs. 13, 14 and 15 show distributions of $\sigma_{22}, \sigma_{12}$ and $\sigma_{11}$ in the entire plate for the case of $h_{1}=0.1$. 


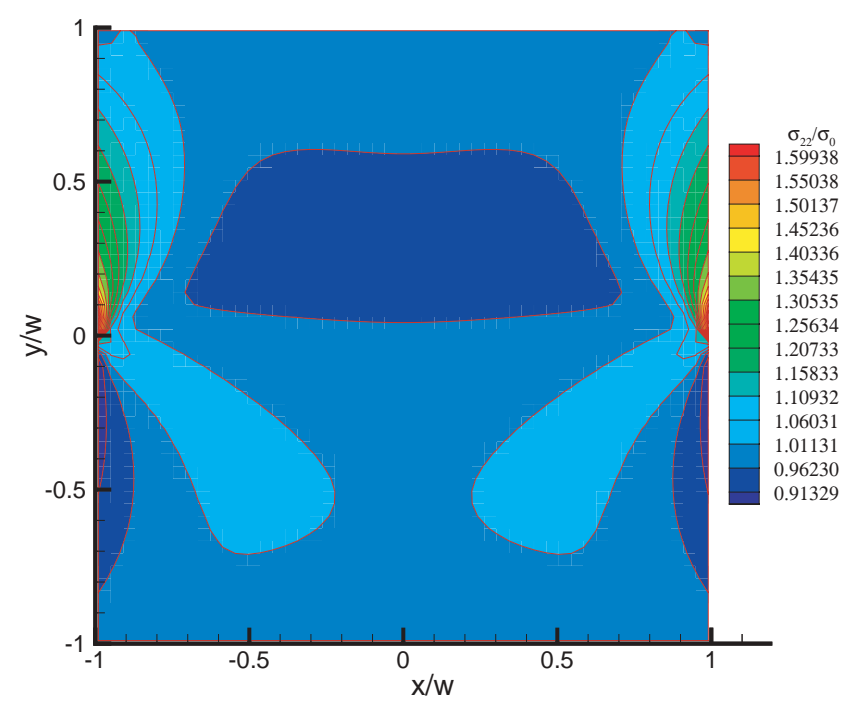

Figure 5: Distribution of $\sigma_{22}$ in an $\mathrm{Al}_{2} \mathrm{O}_{3} / \mathrm{Nb}$ square plate in tension. Thickness of the two layers are the same.

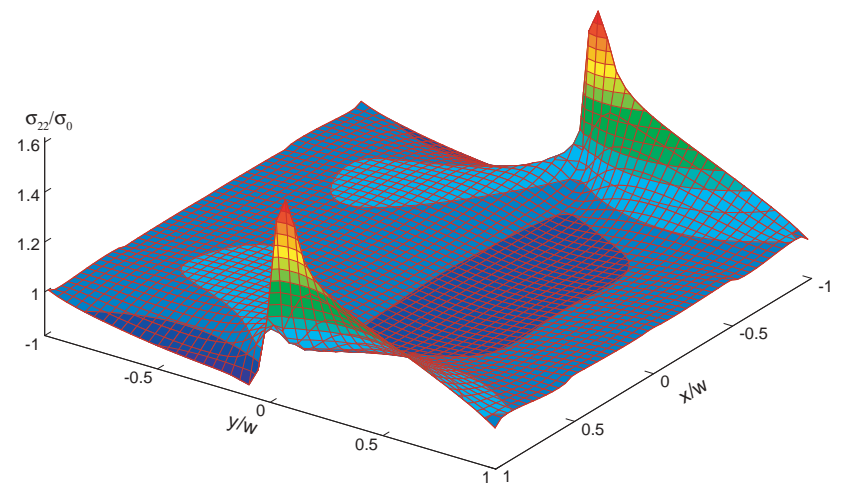

Figure 6: Distribution of $\sigma_{22}$ in an $\mathrm{Al}_{2} \mathrm{O}_{3} / \mathrm{Nb}$ square plate in tension. Thickness of the two layers are the same. 


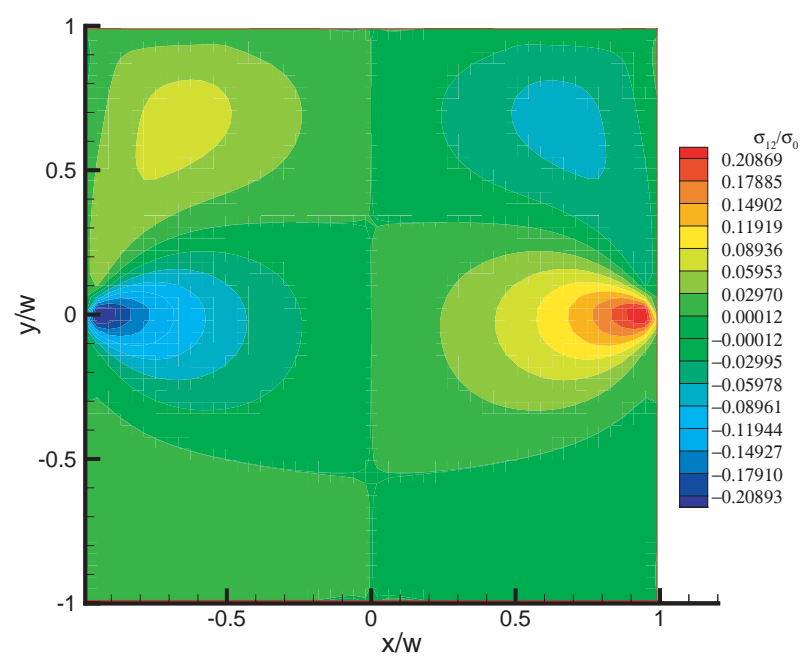

Figure 7: Distribution of $\sigma_{12}$ in an $\mathrm{Al}_{2} \mathrm{O}_{3} / \mathrm{Nb}$ square plate in tension. Thickness of the two layers are the same.

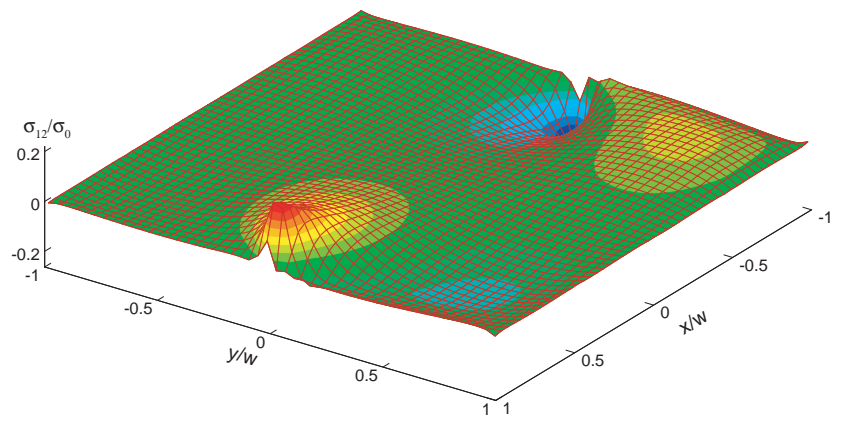

Figure 8: Distribution of $\sigma_{12}$ in an $\mathrm{Al}_{2} \mathrm{O}_{3} / \mathrm{Nb}$ square plate in tension. Thickness of the two layers are the same. 


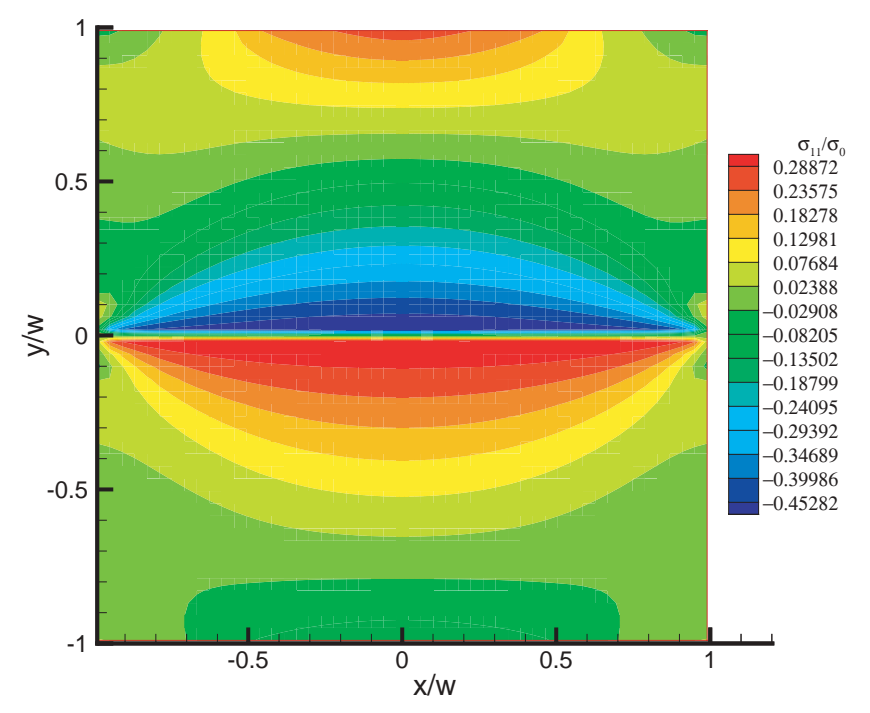

Figure 9: Distribution of $\sigma_{11}$ in an $\mathrm{Al}_{2} \mathrm{O}_{3} / \mathrm{Nb}$ square plate in tension. Thickness of the two layers are the same.

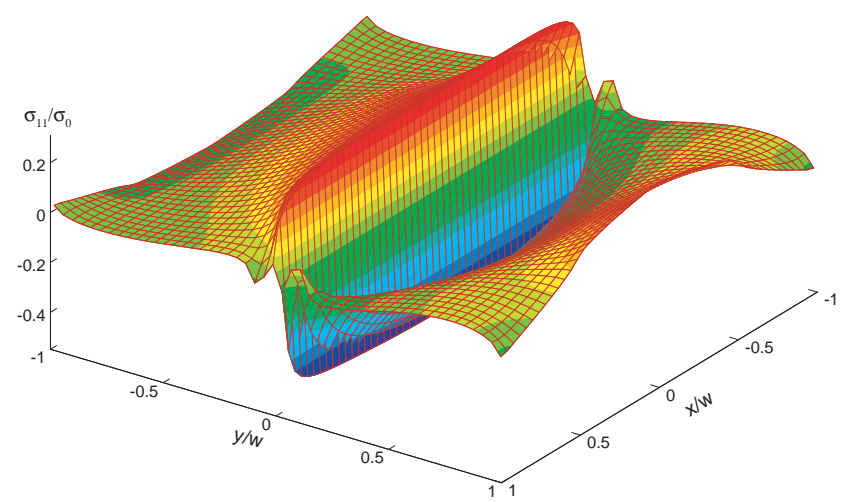

Figure 10: Distribution of $\sigma_{11}$ in an $\mathrm{Al}_{2} \mathrm{O}_{3} / \mathrm{Nb}$ square plate in tension. Thickness of the two layers are the same. 


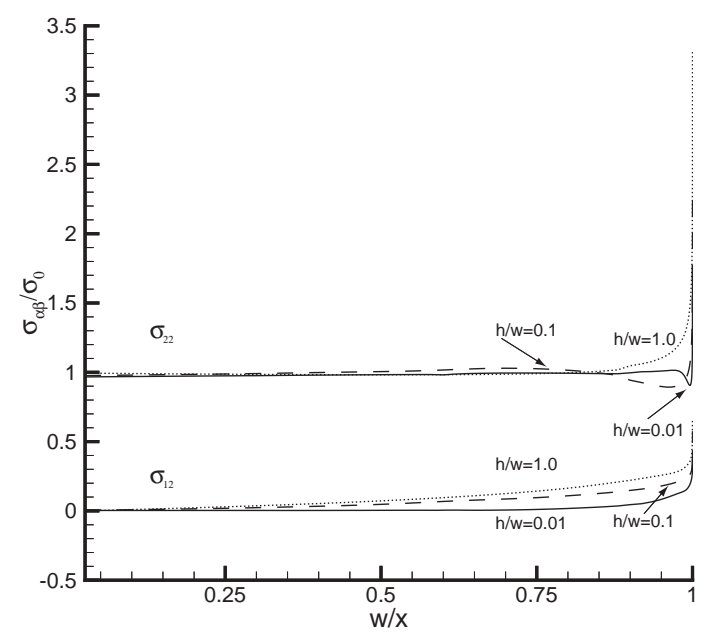

Figure 11: Effect of the $\mathrm{Al}_{2} \mathrm{O}_{3}$ layer thickness $h$ on the interface stress for $\mathrm{Al}_{2} \mathrm{O}_{3}$ $/ \mathrm{Nb}$ bonded systems in tension. Stress components $\sigma_{22}$ and $\sigma_{12}$ are evaluated at $y / w=+1.0 \times 10^{-7}$ for $h_{1}=w$ and $h_{1}=0.1 w$ and at $y / w=+1.0 \times 10^{-10}$ for $h_{1}=0.01 w$.

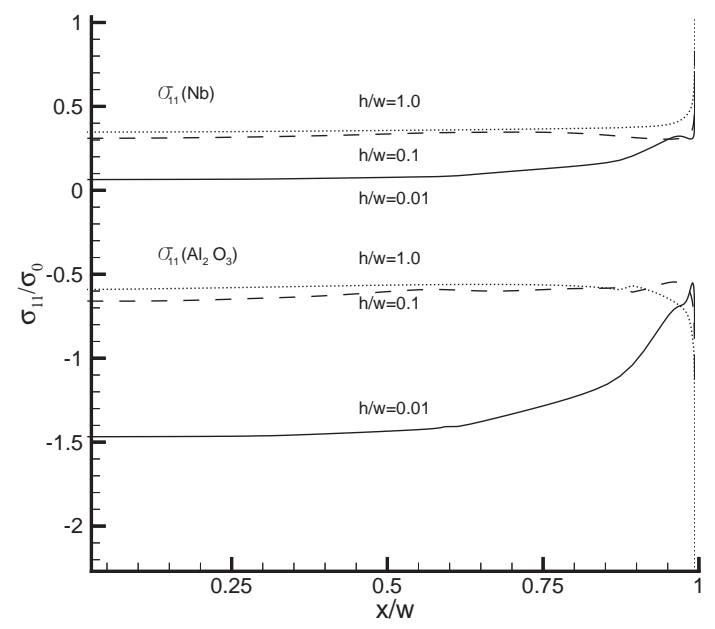

Figure 12: Effect of the $\mathrm{Al}_{2} \mathrm{O}_{3}$ layer thickness $h$ on the interface stress for $\mathrm{Al}_{2} \mathrm{O}_{3}$ / $\mathrm{Nb}$ bonded systems in tension. $\sigma_{11}\left(\mathrm{Al}_{2} \mathrm{O}_{3}\right), \sigma_{11}(\mathrm{Nb})$ are evaluated at $y / w=$ $\pm 1.0 \times 10^{-7}$ for $h_{1}=w$ and $h_{1}=0.1 w$ and at $y / w= \pm 1.0 \times 10^{-10}$ for $h_{1}=0.01 w$. 


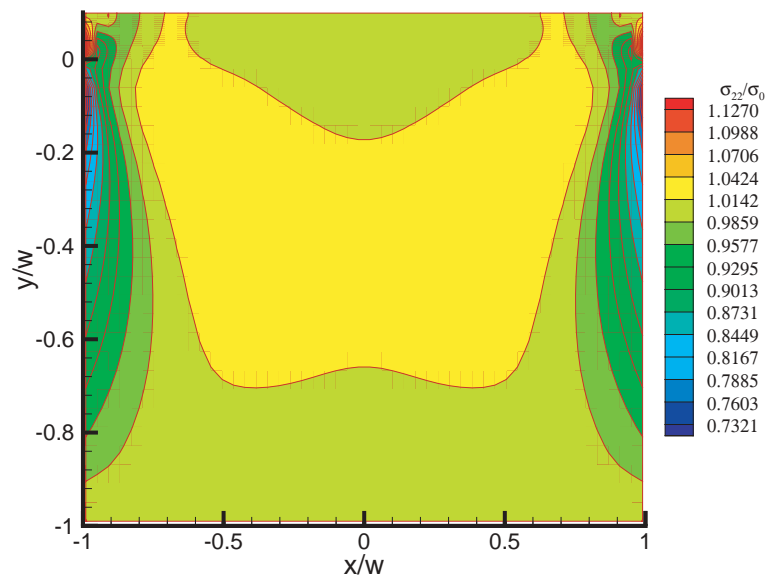

Figure 13: Distribution of $\sigma_{22}$ in an $\mathrm{Nb}$ plate coated with an $\mathrm{Al}_{2} \mathrm{O}_{3}$ layer in tension. Thickness of the coating is $h_{1}=0.1 \mathrm{w}$.

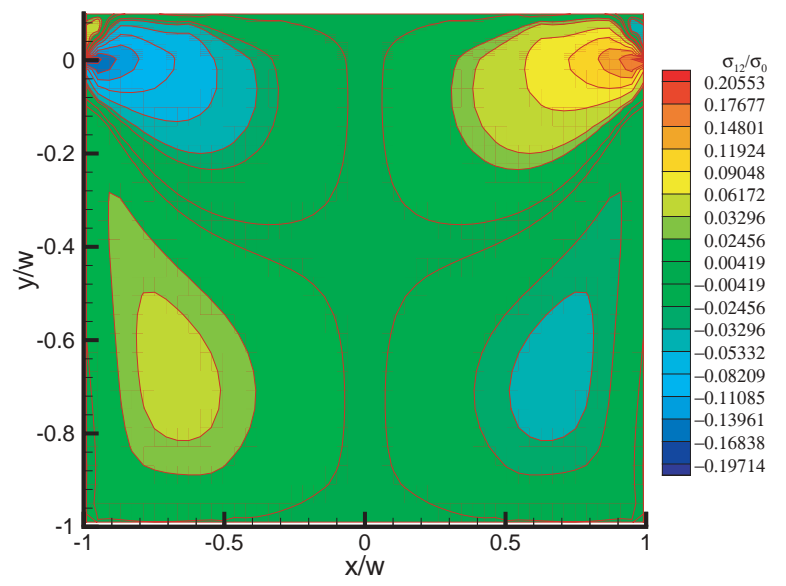

Figure 14: Distribution of $\sigma_{12}$ in an $\mathrm{Nb}$ plate coated with an $\mathrm{Al}_{2} \mathrm{O}_{3}$ layer in tension. Thickness of the coating is $h_{1}=0.1 w$. 


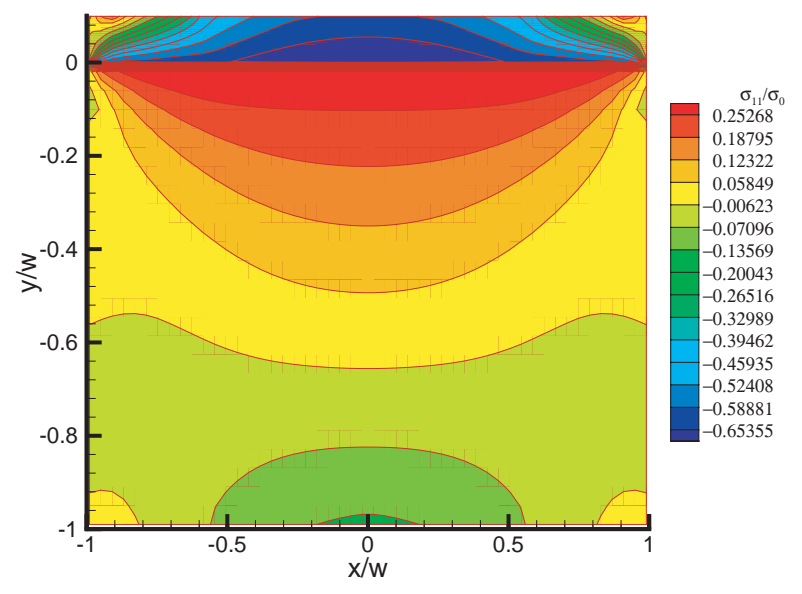

Figure 15: Distribution of $\sigma_{11}$ in an $\mathrm{Nb}$ plate coated with an $\mathrm{Al}_{2} \mathrm{O}_{3}$ layer in tension. Thickness of the coating is $h_{1}=0.1 \mathrm{w}$.

\section{Concluding Remarks}

We have formulated a Green's function BEM for the bimaterial domains with the straight interface. The displacement Green's functions are derived using the complex variable methods in elasticity so that the displacement and traction continuity conditions on the interface are automatically satisfied. There is no need to model the interface. We have applied the BEM to the edge stress concentration problems. We have revealed the accurate details of the edge stress concentration at the free edge of the interface even in the limit of the very thin layers. The application of the BEM for a wider class of bimaterial problems is expected.

\section{References}

[1] J.C.F. Telles and C.A. Brebbia. Boundary element solution for half-plane problems. Int. J. Solids Structures, Vol. 17:pp. 1149-1158, 1981.

[2] J.L. Meek and C. Dai. Boundary element modeling: Near surface excavations. Comp Meth. Appl. Mech. Eng., Vol. 102:pp. 15-27, 1993.

[3] Dumir P.C. and A.K. Mehta. Boundary element solution for elastic orthotropic half-plane problems. Computers and Structures, Vol. 26:pp. 431$438,1987$. 
[4] E. Pan, C.S. Chen, and B. Amadei. A BEM formulation for anisotropic halfplane problems. Engng. Anal. with Boundary Elements, Vol. 20:pp. 185-195, 1997.

[5] M. D. Snyder and T. A. Cruse. Boundary integral equation analysis of cracked anisotropic plates. Int. J. Frac., Vol. 11:pp. 315-328, 1975.

[6] D.L. Clements and M.D. Haselgrove. A boundary integral equation method for a class of crack problems in anisotropic elasticity. Int. J. Comput. Math., Vol. 12:pp. 267-278, 1983.

[7] W.T. Ang. A boundary integral solution for the problem of multiple interacting cracks in an elastic material. Int. J. Fracture, Vol. 31:pp. 259-270, 1986.

[8] M. Morjaria and S. Mukherjee. Numerical analysis of planar time dependent inelastic deformation of plates with cracks by the boundary element method. Int. J. Solids Structures, Vol. 17:pp. 127-143, 1981.

[9] M. Denda and I. Kosaka. Dislocation and point-force-based approach to the special green's function bem for elliptic hole and crack problems in two dimensions. Int. J. Numerical Methods in Engineering, Vol. 40:pp. 2857-2889, 1997.

[10] M. Kamel and B.M. Liaw. Boundary element formulation with special kernels for an anisotropic plate containing an elliptical hole or a crack. Engineering Fracture Mechanics, Vol. 39(4):pp. 695-711, 1991.

[11] C. Hwu and W.J. Yen. Green's functions of two-dimensional anisotropic plates containing an elliptic hole. Int. J. Solids Structures, Vol. 27(13):pp. 1705-1719, 1991.

[12] J. R. Berger. Boundary element analysis of anisotropic bimaterials with special green's functions. Engng. Anal. with Boundary Elements, Vol. 14(2):pp. 123-131, 1994.

[13] R. Yuuki and S.B. Cho. Efficient boundary element analysis of stress intensity factors for interface cracks in dissimilar materials. Engng. Fract. Mech., Vol. 30:pp. 179-188, 1989.

[14] J. R. Berger and V. K. Tewary. Boundary integral equation formulation for interface cracks in anisotropic materials. Int. J. Numerical Methods Engng., Vol. 20:pp. 261-266, 1997.

[15] Y.A. Melnikov. Influence Functions and Matrices. Marcel Dekker, New York, 1999.

[16] N.I. Muskhelishvili. Some basic problems of mathematical theory of elasticity. Noordhoff, Groningen, 1958. 
[17] A.H. England. Complex Variable Methods in Elasticity. Wiley - Interscience, London, 1971.

[18] D.B. Bogy. The plane solution for joined dissimilar elastic semistrips under tension. J. Appl. Mech., Vol. 42:pp. 93-98, 1975.

[19] L. Ni and S. Nemat-Nasser. General duality principle in elasticity. Mech. Materials, Vol. 24(2):pp. 87-123, 1996.

[20] J. Dundurs. Discussion. J. Appl. Mech., Vol. 36:pp. 650-652, 1969.

[21] A.G. Evans, M.C. Lu, S Schmauder, and M Ruhle. Some aspects of the mechanical strength of ceramic/metal bonded systems. Acta metall., Vol. 34(8):pp. 1643-1655, 1986.

\section{Appendix}

\section{A Complex potential functions for the line force and dislocation}

Consider an infinite domain consisting of two semi-infinite blocks of isotropic but different elastic solids with straight interface. Let the $x_{1}$ axis be located on this interface and the elastic moduli of the material occupying the upper $\left(R_{1}\right)$ and the lower $\left(R_{2}\right)$ half planes be $\left(\mu_{1}, \nu_{1}\right)$ and $\left(\mu_{2}, \nu_{2}\right)$, respectively, where $\mu$ and $\nu$ are the shear modulus and Poisson's ratio and the subscripts 1 and 2 identify the materials in $R_{1}$ and $R_{2}$, respectively. Consider a line force $f=f_{x}+i f_{y}$ and a line dislocation with Burgers vector $b=b_{x}+i b_{y}$, respectively, located at $\zeta$ in $R_{1}$. In the complex variable formalism of isotropic elasticity (Muskhelishvili [16] and England [17]) the displacement Green's functions of the line force and dislocation are given by

$$
2 \mu\left(u_{1}+i u_{2}\right)=\kappa \phi(z)-z \overline{\phi^{\prime}(z)}-\overline{\psi(z)},
$$

in terms of analytic functions $\phi(z)$ and $\psi(z)$. To obtain these functions we enforce the continuity of the displacement and the traction along the interface. The application of the standard procedure yields the analytic functions $\left(\phi_{1}, \psi_{1}\right)$ and $\left(\phi_{2}\right.$, $\psi_{2}$ ) in regions $R_{1}$ and $R_{2}$, respectively, according to

$$
\begin{array}{ll}
\phi_{1}(z, \zeta)=\phi_{1 s}(z, \zeta)+\phi_{1 i}(z, \zeta), & \psi_{1}(z, \zeta)=\psi_{1 s}(z, \zeta)+\psi_{1 i}(z, \zeta) \quad\left(z \in R_{1}\right) \\
\phi_{2}(z, \zeta)=\phi_{1 s}(z, \zeta)+\phi_{2 i}(z, \zeta), & \psi_{2}(z, \zeta)=\psi_{1 s}(z, \zeta)+\psi_{2 i}(z, \zeta) \quad\left(z \in R_{2}\right)
\end{array}
$$

where the singular terms

$$
\begin{aligned}
& \phi_{1 s}(z, \zeta)=-\gamma_{1} \log (z-\zeta) \\
& \psi_{1 s}(z, \zeta)=-k_{1} \bar{\gamma}_{1} \log (z-\zeta)+\gamma_{1} \frac{\bar{\zeta}}{z-\zeta}+\gamma_{1},
\end{aligned}
$$


correspond to the solution in the infinite body when the two materials are identical. The image terms

$$
\begin{aligned}
\phi_{1 i}(z, \zeta) & =\delta_{1}\left\{\bar{\psi}_{1 s}(z, \zeta)+z \bar{\phi}_{1 s}^{\prime}(z, \zeta)\right\} \\
\psi_{1 i}(z, \zeta) & =-\delta_{1} z \frac{\partial}{\partial z}\left\{\bar{\psi}_{1 s}(z, \zeta)+z \bar{\phi}_{1 s}^{\prime}(z, \zeta)\right\}+\lambda_{1} \bar{\phi}_{1 s}(z, \zeta) \\
\phi_{2 i}(z, \zeta) & =\lambda_{1} \phi_{1 s}(z, \zeta) \\
\psi_{2 i}(z, \zeta) & =\delta_{1} \psi_{1 s}(z, \zeta)+\left(\delta_{1}-\lambda_{1}\right) z \phi_{1 s}^{\prime}(z, \zeta)
\end{aligned}
$$

represent the modification due to the introduction of a different material in $R_{2}$. The coefficients $\gamma_{1}$ and $k_{1}$ are defined by (7) and $\delta_{1}$ and $\lambda_{1}$ by (9). For a function $\mathcal{F}(z)$ of $z$ we use the notation that

$$
\overline{\mathcal{F}}(z)=\overline{\mathcal{F}(\bar{z})},
$$

such that, for the principal branch of the logarithm, we have

$$
\begin{aligned}
& \bar{\phi}_{1 s}(z, \zeta)=\overline{\phi_{1 s}(\bar{z}, \zeta)}=-\bar{\gamma}_{1} \log (z-\bar{\zeta}) \\
& \bar{\psi}_{1 s}(z, \zeta)=\overline{\psi_{1 s}(\bar{z}, \zeta)}=-k_{1} \gamma_{1} \log (z-\bar{\zeta})+\bar{\gamma}_{1} \frac{\zeta}{z-\bar{\zeta}}+\bar{\gamma}_{1} .
\end{aligned}
$$

The prime attached to a function indicates its derivative with respect to $z$. The solution when the line force and dislocation are in $R_{2}$ can be obtained by interchanging the indices 1 and 2 in the above results.

\section{B Coefficients for constant boundary element}

Let the element $L_{j}$ and the observation point $z_{k}$ be in $R_{1}$, then the coefficients in the system of boundary equations (13) are given by

$$
\begin{aligned}
M_{k j}= & \frac{e^{-i \phi_{j}}}{2 \pi\left(\kappa_{1}+1\right) \mu_{1}}\left[\kappa_{1}\left\{\left\{f_{j}\left(z_{k}\right)+f_{0 j}\left(z_{k}\right)\right\}+e^{2 i \phi_{j}}\left\{\overline{f_{j}\left(z_{k}\right)}+\overline{f_{0 j}\left(z_{k}\right)}\right\}\right\}\right. \\
& \left.-\delta_{1}\left\{\kappa_{1}^{2} e^{2 i \phi_{j}} \bar{f}_{j}\left(z_{k}\right)-(z-\bar{z})\left\{\left(e^{-2 i \phi_{j}}-1\right) s_{j}\left(\bar{z}_{k}\right)+q_{j}\left(\bar{z}_{k}\right)\right\}\right\}-\lambda_{1} f_{j}\left(\bar{z}_{k}\right)\right], \\
N_{k j}= & \frac{e^{-i \phi_{j}}}{2 \pi\left(\kappa_{1}+1\right) \mu_{1}}\left[\left\{e^{-2 i \phi_{j}}\left\{f_{j}\left(z_{k}\right)+f_{0 j}\left(z_{k}\right)\right\}-\left\{h_{j}\left(z_{k}\right)+h_{0 j}\left(z_{k}\right)\right\}\right\}\right. \\
& \left.+\delta_{1} \kappa_{1}\left\{-e^{-2 i \phi_{j}} f_{j}\left(\bar{z}_{k}\right)+w_{j}\left(\bar{z}_{k}\right)-(z-\bar{z}) e^{2 i \phi_{j}} \bar{s}_{j}\left(z_{k}\right)\right\}\right], \\
K_{k j}= & \frac{i}{\pi\left(\kappa_{1}+1\right)}\left[\left\{\kappa_{1}\left\{s_{j}\left(z_{k}\right)+s_{0 j}\left(z_{k}\right)\right\}-\left\{\overline{s_{j}\left(z_{k}\right)}+\overline{s_{0 j}\left(z_{k}\right)}\right\}\right\}\right. \\
& \left.+\delta_{1}\left\{\kappa_{1} \bar{s}_{j}\left(z_{k}\right)+(z-\bar{z}) Q_{j}\left(\bar{z}_{k}\right)\right\}-\lambda_{1} s_{j}\left(\bar{z}_{k}\right)\right], \\
L_{k j}= & -\frac{i}{\pi\left(\kappa_{1}+1\right)}\left[p_{j}\left(z_{k}\right)+\delta_{1}\left\{\kappa_{1} q_{j}\left(\bar{z}_{k}\right)-(z-\bar{z}) \bar{S}_{j}\left(z_{k}\right)\right\}\right] .
\end{aligned}
$$

Let $L_{j}$ be in $R_{1}$ and the observation point $z_{k}$ be in $R_{2}$, then we have

$$
M_{k j}=\frac{e^{-i \phi_{j}}}{2 \pi\left(\kappa_{1}+1\right) \mu_{2}}\left\{\kappa_{2}\left(\lambda_{1}+1\right) f_{j}\left(z_{k}\right)+\kappa_{1}\left(\delta_{1}+1\right) e^{2 i \phi_{j}} \overline{f_{j}\left(z_{k}\right)}\right\},
$$




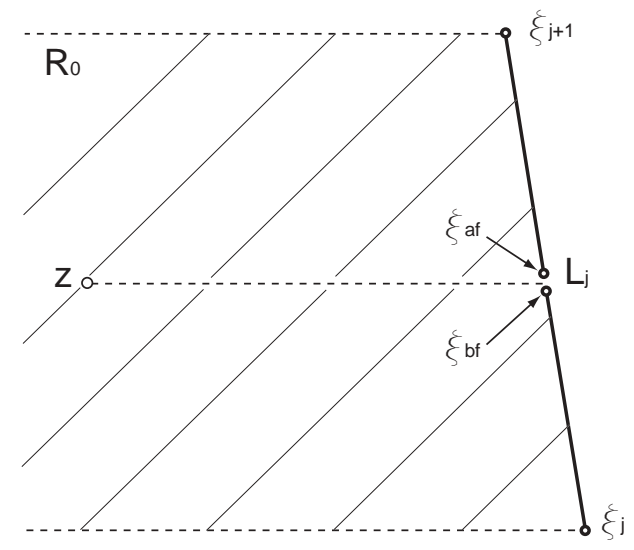

Figure 16: Integration across the branch line of the logarithmic functions.

$$
\begin{aligned}
N_{k j} & =\frac{e^{-i \phi_{j}}}{2 \pi\left(\kappa_{1}+1\right) \mu_{2}}\left[-\left(\delta_{1}+1\right)\left\{-e^{-2 i \phi_{j}} f_{j}\left(z_{k}\right)+w_{j}\left(z_{k}\right)\right\}+\left(\lambda_{1}+1\right)(z-\bar{z}) s_{j}\left(z_{k}\right)\right] \\
K_{k j} & =\frac{i \mu_{1}}{\pi\left(\kappa_{1}+1\right) \mu_{2}}\left\{\kappa_{2}\left(\lambda_{1}+1\right) s_{j}\left(z_{k}\right)-\left(\delta_{1}+1\right) \overline{s_{j}\left(z_{k}\right)}\right\} \\
L_{k j} & =\frac{i \mu_{1}}{\pi\left(\kappa_{1}+1\right) \mu_{2}}\left\{\left(\delta_{1}+1\right) p_{j}\left(z_{k}\right)+\left(\lambda_{1}+1\right)(z-\bar{z}) S_{j}\left(z_{k}\right)\right\}
\end{aligned}
$$

where

$$
\begin{aligned}
s_{j}(z) & =[\log (z-\zeta)]_{\zeta_{j}}^{\zeta_{j+1}}, \quad s_{0 j}(z)=[\log (z-\zeta)]_{\zeta_{a f}}^{\zeta_{b f}}, \\
f_{j}(z) & =[(z-\zeta) \log (z-\zeta)+\zeta]_{\zeta_{j}}^{\zeta_{j+1}}, \quad f_{0 j}(z)=[(z-\zeta) \log (z-\zeta)]_{\zeta_{a f}}^{\zeta_{b f}}, \\
h_{j}(z) & =[\overline{(z-\zeta)} \log (z-\zeta)+\zeta]_{\zeta_{j}}^{\zeta_{j+1}}, \quad h_{0 j}(z)=[\overline{(z-\zeta)} \log (z-\zeta)]_{\zeta_{a f}^{\zeta_{f}}}, \\
w_{j}(z) & =[(z-\bar{\zeta}) \log (z-\zeta)+\zeta]_{\zeta_{j}}^{\zeta_{j+1}}, \quad q_{j}(z)=-\left[\frac{\zeta-\bar{\zeta}}{z-\zeta}\right]_{\zeta_{j}}^{\zeta_{j+1}}, \\
S_{j}(z) & =\left[\frac{1}{z-\zeta}\right]_{\zeta_{j}}^{\zeta_{j+1}}, \quad Q_{j}(z)=-\left[\frac{\zeta-\bar{\zeta}}{(z-\zeta)^{2}}\right]_{\zeta_{j}}^{\zeta_{j+1}}, \quad P_{j}(z)=\left[\frac{\overline{z-\zeta}}{(z-\zeta)^{2}}\right]_{\zeta_{j}}^{\zeta_{j+1}} .
\end{aligned}
$$

Note that terms $s_{0 j}(z), f_{0 j}(z)$ and $h_{0 j}(z)$ appear only when the observation point $z$ is located to the left of the boundary element $L_{j}$ (region $R_{0}$ in Fig. 16). This is caused by the jump in the argument of the logarithm as the source point $\zeta$ moves along $L_{j}$ during the integration. When this jump occurs the integration has to be stopped before the jump (i.e. at $\zeta_{b f}$ ) and then be restarted after the jump (i.e. at $\left.\zeta_{a f}\right)$ as shown in Fig. 16. The results when the element $L_{j}$ is located in $R_{2}$ are obtained from the above by interchanging the indices 1 and 2 . 